\title{
Mamíferos silvestres de la Reserva Ecológica del Pedregal de San Ángel en Ciudad Universitaria, Universidad Nacional Autónoma de México, México, D. F.
}

\author{
Wild mammals of Reserva Ecológica del Pedregal de San Ángel, Ciudad Universitaria, UNAM, \\ Mexico, D.F.
}

\author{
Yolanda Hortelano-Moncada*, Fernando A. Cervantes y Aída Trejo-Ortiz \\ Departamento de Zoología, Instituto de Biología, Universidad Nacional Autónoma de México. Apartado postal 70-153, 04510 México, D. F.,México. \\ *Correspondencia: yolahm@ibiologia.unam.mx
}

\begin{abstract}
Resumen. Se realizó un inventario de las especies de mamíferos que habitan en la Reserva Ecológica del Pedregal de San Ángel (REPSA). Los datos se obtuvieron a partir de registros de colecciones científicas, de recolectas recientes en el área y de consultas en literatura especializada. Los resultados comprenden 628 registros acumulados desde 1943 y corresponden a 33 especies, agrupadas en 28 géneros, 15 familias y 6 órdenes de mamíferos. La ardilla gris (Sciurus aureogaster nigrescens) y el ratón del altiplano (Peromyscus melanophrys melanophrys) se registran por primera vez para la REPSA; asimismo, existen 2 registros que están publicados pero no listados en los inventarios previos a la reserva, el murciélago colorado, Lasiurus blossevillii teliotis, y el cuinique, Spermophilus adocetus adocetus; en las categorías de riesgo se encuentran como amenazadas 2 especies y 1 en la de protección especial, y hay 7 endémicas de México. La Reserva es uno de los últimos reductos de material genético de especies cuya localidad tipo se encuentra en la cuenca de México. El componente mastofaunístico de la zona es importante para el mantenimiento de la biodiversidad, por lo que el estudio y la protección de este ecosistema al interior de la ciudad de México debe continuarse.
\end{abstract}

Palabras clave: mamíferos, ciudad de México, colecciones biológicas, inventarios, Reserva del Pedregal.

\begin{abstract}
This paper documents the mammals from the Reserva Ecológica del Pedregal de San Ángel. Data were gathered from records in mammal collections, the specialized literature, and through collecting efforts in the area. Records spanning 1943 to the present document the presence of 33 species, 28 genera, 15 families, and 6 orders of mammals. One squirrel and one mouse (Sciurus aureogaster nigrescens, and Peromyscus m. melanophrys) are reported for the first time for the reserve. Two addtional records have been previously published but have not been included in previous inventories for the reserve: the red bat, Lasiurus blossevillii teliotis and the cuinique, Spermophilus adocetus adocetus. Two species are currently listed under the risk category of threatened, 1 is under special protection, and 7 are endemic to Mexico. This mammal component is important for the ecosystem of the area and the conservation of biodiversity. The richness of mammals of the area deserves efforts to conserve and preserve the biodiversity of this unique ecosystem within México City.
\end{abstract}

Key words: mammals, México City, biological collections, inventory, Reserva del Pedregal.

\section{Introducción}

La Reserva Ecológica del Pedregal de San Ángel (REPSA) se encuentra al suroeste de la ciudad de México, en los terrenos de Ciudad Universitaria, por lo que es custodiada y manejada por la Universidad Nacional Autónoma de México. La REPSA tiene un alto valor biológico y cultural para la conservación de la biodiversidad y representa un patrimonio importante del Distrito Federal. Esta área es uno de los pocos ecosistemas naturales del sur de la Cuenca de México y destaca por

Recibido: 14 diciembre 2007; aceptado: 31 enero 2009 sus características físicas, químicas y biológicas muy particulares que ameritan ser conservadas (Rojo, 1994; de la Fuente, 2005). Recientemente las medidas para manejar esta reserva condujeron a incrementar su área (de 146.8 a 237.3 ha), rezonificarla y definir sus límites (de la Fuente, 2005; Molina y Peralta-Higuera, 2005). Al mismo tiempo se aplicaron acciones para su protección y vigilancia con la finalidad de asegurar la conservación de este patrimonio. A fin de ayudar a cumplir con este propósito se han efectuado estudios recientes para conocer la diversidad biológica de la REPSA (Herrera et al., 2006). Desafortunadamente, el inventario de la fauna está incompleto y la información disponible es escasa y aislada. En consecuencia, con los 
datos disponibles resulta difícil definir planes de manejo y acciones de conservación adecuados para la protección y el aprovechamiento del área.

Los primeros registros de mamíferos en el área de la Reserva surgieron de evaluaciones mastofaunísticas globales sobre Norteamérica y la Cuenca de México (Hall y Kelson, 1959; Hall, 1981, Villa, 1952, 1966; Ceballos y Galindo, 1984; Sánchez et al., 1989). Asimismo, algunos autores efectuaron recopilaciones bibliográficas (Álvarez et al., 1994) de nombres de especies vegetales y animales del área en apoyo de los esfuerzos para la conservación de la zona natural que después sería el área de la Reserva. Los resultados incluyeron un listado de especies de mamíferos. Paralelamente, se realizaron otros estudios en los que se evaluó la identidad de algunas especies de mamíferos y aspectos de su distribución, abundancia y estado actual de sus poblaciones (Negrete, 1991, Negrete et al., 1994). Estos esfuerzos corroboraron la presencia de 26 especies de las 37 registradas hasta ese momento, las restantes las consideraron ausentes de la REPSA. Desafortunadamente, los resultados no se documentaron con la preparación de ejemplares de museo, el registro en catálogos o algúna otra forma, lo que hace imposible verificar dichos resultados. Ello, por lo tanto, dificulta la consulta y el acopio de información sobre los mamíferos presentes en la REPSA. Otros estudios también incluyeron registros de los mamíferos silvestres del Pedregal enfocándose en los roedores (Chávez, 1993 a y b; Chávez y Ceballos, 1992; Chávez y Ceballos, 1994). Su resultado corrobora la presencia de 6 especies en el área y algunos aspectos de su historia natural, aunque los autores tampoco documentaron la identidad taxonómica de las especies observadas.

Es necesario, entonces, generar información básica y documentada sobre el componente mastozoológico de la biodiversidad del área que proporcione herramientas para un programa adecuado de conservación de la Reserva. Por lo tanto, el propósito de este trabajo es registrar el inventario actualizado de los mamíferos de la REPSA. Desde luego, es indispensable actualizar la nomenclatura y corroborar la identidad taxonómica de los ejemplares de museo del área en cuestión que están depositados en las colecciones biológicas.

\section{Materiales y métodos}

El área de estudio fue la Reserva Ecológica del Pedregal de San Ángel que se localiza en Ciudad Universitaria, campus de la Universidad Nacional Autónoma de México, y zonas circundantes. El área de la Reserva tiene 237.3 ha (una zona núcleo de 171 ha y una de amortiguamiento de 66 ha) y se encuentra en la delegación Coyoacán, al suroeste del Distrito Federal (entre $19^{\circ} 20^{\prime} 22^{\prime}$ ' y $19^{\circ} 13^{\prime}$ 25' ' N y 99० 8' 26', y 99 14' 3', O, a $2200-2277$ $\mathrm{m}$ sobre el nivel del mar). El sustrato de esta superficie es típicamente basáltico, producto de la erupción del volcán Xitle, hace aproximadamente 2500 años, y de la accidentada topografía. Su clima es templado subhúmedo con lluvias en verano y la temperatura es de 14 a $15^{\circ} \mathrm{C}$, con precipitación media anual de 700 a $900 \mathrm{~mm}$ (de la Fuente, 2005). Debido a la variabilidad del sustrato y altitud, la Reserva presenta diferentes asociaciones vegetales, siendo ésta clasificada como matorral xerófilo, con dominancia de arbustos (Senecio praecox) y hierbas. Algunos árboles exóticos, como el eucalipto (Eucalyptus sp.) y el pirú (Schinus molle), son ahora abundantes.

Para obtener el inventario actualizado de la REPSA se realizaron diversas actividades. Se revisó cuidadosamente la literatura para obtener los registros de especies de mamíferos en localidades al interior de la REPSA, y se buscó la fuente bibliográfica original para verificar la información.

Debido a la vecindad de la REPSA con las instalaciones del Instituto de Biología, UNAM, los autores efectuaron recolectas ocasionales en el área de estudio con relación a otros proyectos, para las que se utilizaron redes de niebla, trampas Sherman y Tomahawk. Asimismo, durante el desarrollo de este trabajo los autores recuperaron del área de estudio mamíferos muertos, principalmente tlacuaches y ardillas, la mayoría de ellos atropellados por vehículos en las vías de circulación de Ciudad Universitaria. Todos los ejemplares se prepararon como ejemplares de museo y fueron determinados taxonómicamente siguiendo los procedimientos convencionales (Hall, 1981; Medellín et al., 1997; Villa y Cervantes, 2003). Los datos de estas localidades se registraron con precisión, usando un geoposicionador (marca Garmin) para el cálculo de las coordenadas geográficas. El material resultante de las recolectas se depositó y catalogó en la Colección Nacional de Mamíferos (CNMA).

Por otro lado, se consultaron bases de datos y ejemplares depositados en las siguientes colecciones de la ciudad de México: Colección Nacional de Mamíferos, Instituto de Biología, UNAM (CNMA), Colección de Cordados, Escuela Nacional de Ciencias Biológicas, Instituto Politécnico Nacional (ENCB), Museo de Zoología "Alfonso L. Herrera", Facultad de Ciencias, UNAM (MZFC) y Universidad Autónoma MetropolitanaIztapalapa (UAMI).

Para complementar la información anterior, se consultaron por Internet los bancos de datos de la Unidad de Informática para la Biodiversidad (UNIBIO, 2006), y Mammal Networked Information System,MANIS (Wieczorek, 2001), en otros casos, se solicitó la base de 
datos a los responsables de los acervos o se tomaron los datos directamente de los ejemplares y catálogos asociados a ellos.

Se encontraron registros de ejemplares de museo de especies de mamíferos mexicanos en las siguientes 16 colecciones mastozoológicas: San Diego Natural History Museum (SDNHM), Utah Museum of Natural History (UMNH), University of Washington Burke Museum (UWBM), California Academy of Sciences (CAS), Bishop Museum of Natural History (BPBM), The Museum, Texas Tech University (TTU), Museum of Southwestern Biology, University of New Mexico (MSB), Los Angeles County Museum of Natural History (LACM), Royal Ontario Museum (ROM), University of Colorado Museum (UCM), University of Alaska Museum of the North (UAM), Natural History Museum, University of Kansas (KU), Field Museum of Natural History (FMNH), Museum of Zoology, University of Michigan (UMMZ), Museum of Vertebrate Zoology, University of California, Berkeley (MVZ) y Louisiana State University Museum of Natural Science (LSUMZ). Los acrónimos de las Colecciones se tomaron de acuerdo con Hafner et al. (1997).

Para su manejo y análisis, se elaboró una base de datos con el programa Microsoft Access 2003, donde se incorporó la información biológica y la geográfica de los ejemplares en 50 campos, de acuerdo con los lineamientos del Darwin Core (Ver. 3.0) y de MaNIS/HerpNet/ORNIS Georeferencing Guidelines (Wieczorek, 2001). Debido a que algunas localidades de colecta de los ejemplares depositados en las colecciones eran incorrectas o su nombre era ambiguo, fue necesario revisar literatura científica, diarios y catálogos de campo para ubicar o verificar la localidad geográfica mencionada.

Los nombres de las localidades se estandarizaron y se calcularon sus coordenadas geográficas empleando una guía para georreferenciar (Wieczorek, 2001); se utilizaron listados de localidades y cartas topográficas a escalas de 1:50,000 ó 1:100,000 (INEGI,, 2001; SCT, 1987, respectivamente); en algunos casos, se tomaron datos directamente con un geoposicionador (marca Garmin.). La información obtenida de la georreferenciación se visualizó utilizando el software Arcview y el mapa digitalizado de la REPSA (Fig. 1) preparado por el Instituto de Geografía, UNAM (Peralta et al., 2005; Molina y Peralta-Higuera, 2005).

Se actualizó la nomenclatura y clasificación de los nombres de los mamíferos que se encontraron en la REPSA de acuerdo con el listado taxonómico de Ramírez-Pulido et al. (2005), con excepción de la familia Heteromyidae, para la cual se siguieron las recomendaciones de Hafner et al. (2007).

\section{Resultados}

La base de datos que se elaboró a partir de los registros de las colecciones así como de los ejemplares recolectados produjo un total de 628 registros de mamíferos dentro del perímetro de la REPSA. La piel, cráneo y esqueleto de los ejemplares se encuentran preservados en alcohol; fueron recolectados en el transcurso de 64 años (1943 - 2007) y se encuentran depositados en 8 colecciones mastozoológicas cuyos acrónimos se anotan a continuación seguidos, entre paréntesis, por el número de ejemplares que se encontró en cada una de ellas: CNMA (297), UMMZ (151), ENCB (52), FMNH (43), KU (36), MVZ (34), MZFC (10) y UAMI (5). La base de datos está disponible en el Portal UNIBIO (http//unibio.ibunam.mx).

Los resultados destacan que el mayor número de ejemplares (303) se recolectó entre 1943 y 1949, seguido por los de las décadas de 1960 (77) y 1980 (49). El número de ejemplares recolectados en un año varió de 1 a 151 y se registraron periodos de hasta 5 años sin recolectas, lo que indica que existe un porcentaje pequeño de registros que se obtuvo de manera incidental. Asimismo, los resultados muestran que contribuyeron unos 50 recolectores.

El número total de especies registradas en este estudio para la REPSA fue de 33 (Cuadro 1), correspondientes a 28 géneros, 15 familias y 6 órdenes, en el cual están incluidos 2 nuevos registros para el área de estudio: la ardilla gris (Sciurus aureogaster nigrescens), y el ratón del altiplano (Peromyscus melanophrys).

El primer ejemplar de $S$. aureogaster nigrescens que se colectó en la REPSA corresponde a 1999 (CNMA 39693). Probablemente esta ardilla es una especie de reciente introducción ya que no se le había nombrado en publicaciones anteriores y en la actualidad es abundante en la Reserva y sus alrededores. El otro registro es de $P$. m. melanophrys, un macho recolectado el 26 de junio de 1984, en el "Pedregal de San Ángel” [sic]), con número de catálogo CNMA 32015, sólo cráneo. Para el Distrito Federal hay un registro cercano de esta especie que corresponde a Contreras (Baker, 1952).

Los órdenes mejor representados son Rodentia con el $40 \%$ (13 especies) y Chiroptera con el $40 \%$ (12 especies), seguidos de Carnivora con el $15 \%$ (5 especies). Los órdenes Soricomorpha, Didelphimorphia y Lagomorpha están representados con el 3\% cada uno, lo que corresponde a una especie por orden. La distribución espacial de estas especies comprende el área de la REPSA (zona núcleo y de amortiguamiento), el campus de Ciudad Universitaria y zonas aledañas, San Jerónimo, Pedregal de San Ángel, Tizapán y Coyoacán (Fig. 1, Cuadro 3). La representación de ejemplares por especie encontrada en las colecciones mastozoológicas varía de 1 a 316 (Apéndice 


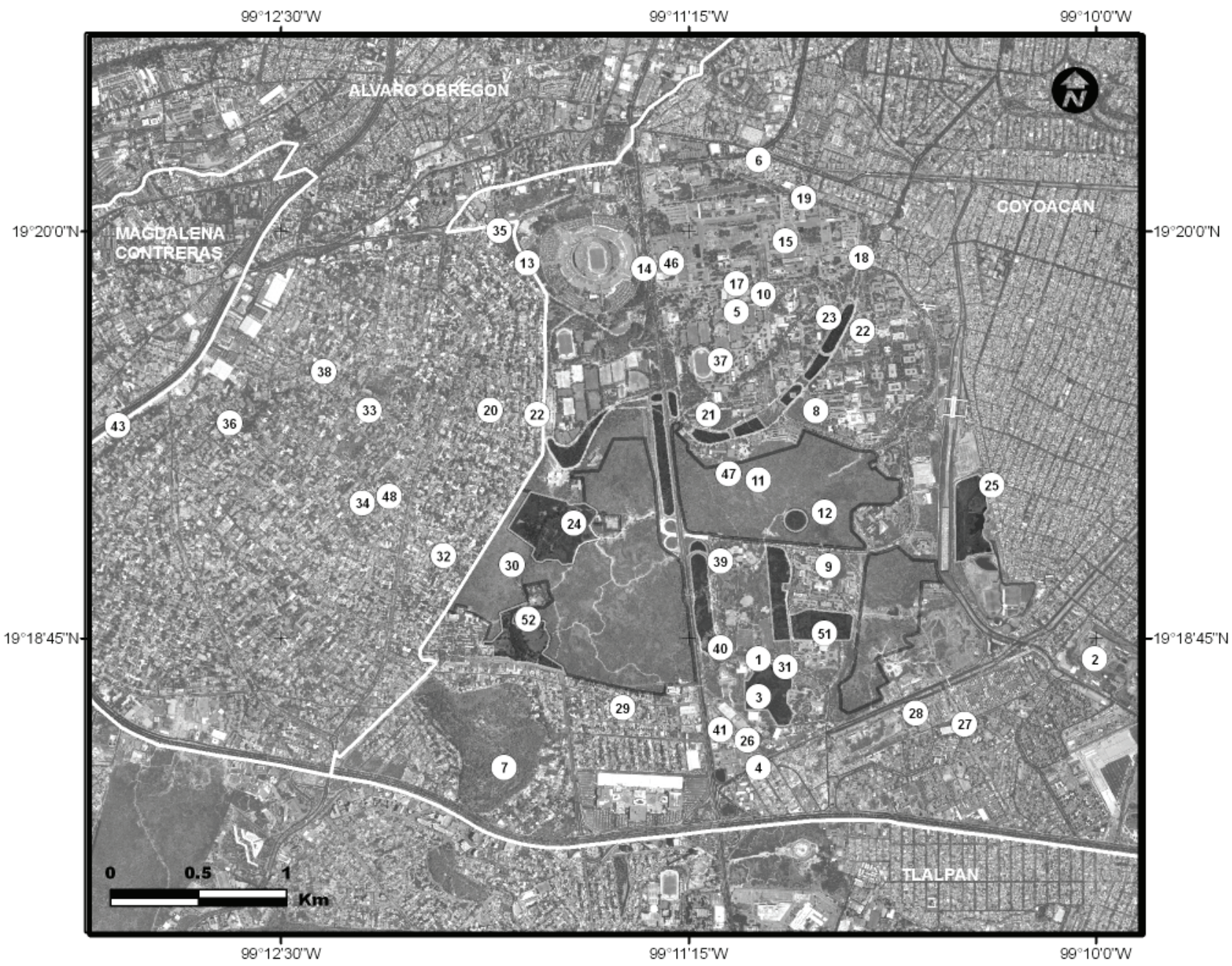

Figura 1. Posición geográfica de las localidades de recolecta (Cuadro 3) de los registros de ejemplares de museo de mamíferos en la Reserva Ecológica del Pedregal de San Ángel, Ciudad Universitaria, Universidad Nacional Autónoma de México en la , ciudad de México. La zona núcleo está representada por los polígonos claros y la de amortiguamiento por los obscuros.

1). Con un número de ejemplares relativamente alto están representados 4 taxa (12\%): el tlacuache (Didelphis virginiana), el ardillón (Spermophilus variegatus), el ratón pigmeo (Baiomys taylori) y el ratón piñonero (Peromyscus gratus), con 41, 24, 69 y 316 ejemplares, respectivamente. En contraste, se registraron 11 especies (32\%) que están representadas sólo por un ejemplar; tal es el caso de la ardilla cuinique (S. a. adocetus), el ratón del altiplano ( $P$. m. melanophrys), la tuza llanera (Cratogeomys merriami merriami), la zorra gris (Urocyon cineroargenteus nigrirostris), la comadreja (Mustela frenata frenata), y varios murciélagos (L. blosevilli teliotis, Idionycteris phyllotis, Eptesicus fuscus y Myotis velifer).

De las especies de mamíferos registradas para la REPSA, 3 se encuentran en alguna categoría de riesgo definida por la Norma Oficial Mexicana NOM-059-ECOL2001 (SEMARNAT, 2002). En categoría de "amenazada" están 2 murciélagos filostómidos: Leptonycteris curasoae y Choeronycteris mexicana, mientras que un murciélago vespertiliónido, Corynorhinus mexicanus, se encuentra en categoría de "protección especial" (Cuadro 1).

Los mamíferos de la REPSA incluyen 5 taxa endémicos de México, 2 especies de ratones (P. g. gratus y P. m. melanophrys), 1 tuza (C. m. merriami), 1 ardilla $(S$. a. adocetus) y 1 murciélago (C. mexicanus); asimismo, se documentaron 4 especies monotípicas, conformadas por 1 roedor (Sigmodon hispidus) y 3 murciélagos (C. mexicana, Nyctinomops macrotis y Corynorhinus mexicanus (Cuadro 1). Ninguna especie es endémica de la REPSA, del Distrito Federal o de la Cuenca de México. 
Cuadro 1. Mamíferos de la Reserva Ecológica del Pedregal de San Ángel en Ciudad Universitaria, Universidad Nacional Autónoma de México (REPSA)

\begin{tabular}{lll}
\hline Especies & Categoría de riesgo Endémico \\
\hline
\end{tabular}

Clase Mammalia

Infraclase Metatheria

Subclase Theriformes

Orden Didelphimorphia

Familia Didelphidae

Subfamilia Didelphinae

Didelphis virginiana californica

Orden Lagomorpha

Familia Leporidae

Sylvilagus floridanus orizabae

Orden Rodentia

Familia Sciuridae

Subfamilia Sciurinae

Sciurus aureogaster nigrescens*

Spermophilus adocetus adocetus

$\mathrm{X}$

Spermophilus variegatus variegatus

Familia Muridae

Subfamilia Sigmodontinae

Baiomys taylori analogus

Neotoma mexicana torquata

Peromyscus gratus gratus

Peromyscus maniculatus labecula

Peromyscus melanophrys melanophrys*

Reithrodontomys fulvescens toltecus

Reithrodontomys megalotis saturatus

Sigmodon hispidus

Familia Geomyidae

Subfamilia Geomyinae

Cratogeomys merriami merriami

Familia Heteromyidae

Heteromys irroratus alleni

Orden Carnivora

Familia Canidae

Subfamilia Caninae

Urocyon cinereoargenteus nigrirostris

Familia Mephitidae.

Mephitis macroura macroura

Spilogale putorius angustifrons

Familia Procyonidae

Subfamilia Bassariscinae

Bassariscus astutus astutus

Familia Mustelidae

Subfamilia Mustelinae

Mustela frenata frenata

Orden Soricomorpha

Familia Soricidae

Subfamilia Soricinae

Sorex saussurei saussurei 
Cuadro 1. Continúa

\begin{tabular}{|c|c|c|c|}
\hline Especies & Categoría de riesgo & Endémico & Monotípico \\
\hline \multicolumn{4}{|l|}{$\begin{array}{l}\text { Orden Chiroptera } \\
\text { Familia Mormoopidae } \\
\quad \text { Mormoops megalophylla megalophylla }\end{array}$} \\
\hline $\begin{array}{l}\text { Familia Phyllostomidae } \\
\text { Subfamilia Glossophaginae } \\
\text { Leptonycteris curasoae yerbabuenae } \\
\text { Anoura geoffroyi lasiopyga } \\
\text { Choeronycteris mexicana }\end{array}$ & $\begin{array}{l}\text { Amenazada } \\
\text { Amenazada }\end{array}$ & & $X$ \\
\hline $\begin{array}{l}\text { Familia Molossidae } \\
\text { Subfamilia Molossinae } \\
\text { Tadarida brasiliensis mexicana } \\
\text { Nyctinomops macrotis }\end{array}$ & & & $X$ \\
\hline $\begin{array}{l}\text { Familia Vespertilionidae } \\
\text { Subfamilia Vespertilioninae } \\
\text { Lasiurus blossevilli teliotis** } \\
\text { Lasiurus cinereus cinereus } \\
\text { Corynorhinus mexicanus } \\
\text { Idionycteris phyllotis } \\
\text { Eptesicus fuscus miradorensis }\end{array}$ & & $X$ & $\mathrm{X}$ \\
\hline $\begin{array}{l}\text { Subfamilia Myotinae } \\
\text { Myotis velifer velifer }\end{array}$ & Protección especial & & \\
\hline
\end{tabular}

Fuentes: Ramírez-Pulido et al., 2005; Hafner et al., 2007; para las categorías de riesgo SEMARNAT, 2002.

* Mamíferos registrados por primera vez para esta área.

** Registros publicados, pero no enlistados en los inventarios previos.

En la literatura científica se registran 13 holotipos de mamíferos, correspondientes a 5 localidades tipo en el Distrito Federal (Álvarez et al., 1997). De estas especies, 9 son o fueron habitantes de la Reserva; tal es el caso de Mustela frenata, Bassariscus astutus, Mephitis macroura, Spilogale putorius, Peromyscus gratus, Reithrodontomys fulvescens, Liomys irroratus y Nyctinomops macrotis. Esto es de gran importancia, ya que en algunos casos la localidad tipo ha sido sustituida por asentamientos humanos; por lo tanto, las especies podrían no estar ya en las zonas donde fueron descritas originalmente, siendo la Reserva el último reducto de material genético de estas especies.

Por otro lado, en la REPSA habitan también mamíferos exóticos y ferales, como: la rata de caño (Rattus norvegicus), el ratón doméstico (Mus musculus), el gato doméstico (Felis silvestres) y el perro doméstico (Canis familiaris), pero que no fueron considerados en la lista de resultados

\section{Discusión}

En la revisión bibliográfica se identificaron 15 referencias, donde se mencionan registros de mamíferos para la REPSA (Álvarez et al. 1994; Castellanos, 2006; Ceballos y Galindo, 1984; Chávez, 1993a y b; Chávez y Ceballos, 1992; Chávez y Ceballos, 1994; García, 2007; Hall, 1981; Negrete y Soberón, 1994, Sánchez et al., 1989; Villa, 1952; Villa, 1966; Villa y Sánchez 1991; Villa y Cervantes, 2003). Encontramos que 8 de estas publicaciones son exclusivas de mamíferos de la REPSA y el resto son evaluaciones globales de la Cuenca de México, del país o de Norteamérica (Cuadro 2). El trabajo más antiguo en donde se hace referencia a mamíferos de la REPSA es de hace 56 años (Villa, 1952) y el más reciente corresponde a García (2007).

El número de especies silvestres registrado para la REPSA en cada referencia es variable, pero el total acumulado hasta antes del presente estudio y con la nomenclatura actualizada, según Ramírez-Pulido et al. (2005) es de 37, sin considerar las especies exóticas, como los roedores Rattus rattus, Rattus norvegicus y Mus musculus. Los registros extraídos para la REPSA de publicaciones para la Cuenca de México o para el país, oscilaron entre 1 y 37. 
Cuadro 2. Registros bibliográficos de la mastofauna silvestre de la Reserva Ecológica del Pedregal de San Ángel, Ciudad Universitaria, Universidad Nacional Autónoma de México

\begin{tabular}{|c|c|c|c|c|c|c|c|c|c|c|c|c|}
\hline Especie & Sinónimos & 1 & 2 & 3 & 4 & 5 & 6 & 7 & 8 & 9 & 10 & $P E$ \\
\hline Didelphis virginiana californica & & $\mathrm{X}$ & & & $\mathrm{X}$ & & $\mathrm{X}$ & $\mathrm{X}$ & & $\mathrm{X}$ & & $\mathrm{X}$ \\
\hline Sylvilagus floridanus orizabae & S. orizabae $e^{9}$ S. floridanus ${ }^{9}$ & $\mathrm{X}$ & & & $\mathrm{X}$ & & $\mathrm{X}$ & & & $\mathrm{X}$ & & $\mathrm{X}$ \\
\hline *Sciurus aureogaster nigrescens & & & & & & & & & & & & $\mathrm{X}$ \\
\hline **Spermophilus adocetus adocetus & & & & & & & & & & & & $\mathrm{X}$ \\
\hline ***Spermophilus mexicanus mexicanus & & & & & $\mathrm{X}$ & & $\mathrm{X}$ & & & $\mathrm{X}$ & & \\
\hline Spermophilus variegatus variegatus & & & & & $\mathrm{X}$ & & $\mathrm{X}$ & $\mathrm{X}$ & $\mathrm{X}$ & $\mathrm{X}$ & & $\mathrm{X}$ \\
\hline ***Microtus mexicanus mexicanus & & & & & & & $\mathrm{X}$ & & & $\mathrm{X}$ & & \\
\hline Baiomys taylori analogus & & $\mathrm{X}$ & & & $\mathrm{X}$ & & $\mathrm{X}$ & & & $\mathrm{X}$ & & $\mathrm{X}$ \\
\hline Neotoma mexicana torquata & & & & & $\mathrm{X}$ & & $\mathrm{X}$ & $\mathrm{X}$ & $\mathrm{X}$ & $\mathrm{X}$ & & $\mathrm{X}$ \\
\hline Peromyscus gratus gratus & P. truei gratus $^{9}$, P. gratus $^{8}$ & $\mathrm{X}$ & & & $\mathrm{X}$ & & $\mathrm{X}$ & $\mathrm{X}$ & $\mathrm{X}$ & $\mathrm{X}$ & & $\mathrm{X}$ \\
\hline Peromyscus maniculatus labecula & & & & & & & $\mathrm{X}$ & & & $\mathrm{X}$ & & $\mathrm{X}$ \\
\hline *Peromyscus melanophrys melanophrys & & & & & & & & & & & & $\mathrm{X}$ \\
\hline$* * *$ Peromyscus melanotis & & $\mathrm{X}$ & & & $\mathrm{X}$ & & $\mathrm{X}$ & & & & & \\
\hline Reithrodontomys fulvescens toltecus & & $\mathrm{X}$ & & & & & $\mathrm{X}$ & $\mathrm{X}$ & $\mathrm{X}$ & $\mathrm{X}$ & & $\mathrm{X}$ \\
\hline Reithrodontomys megalotis saturatus & & $\mathrm{X}$ & & & $\mathrm{X}$ & & $\mathrm{X}$ & & & $\mathrm{X}$ & & $\mathrm{X}$ \\
\hline Sigmodon hispidus & $\begin{array}{l}\text { S. } h . \text { berladieri }{ }^{4,6,7,10}, S . h \text {. } \\
\text { obvelatus }\end{array}$ & & & & $\mathrm{X}$ & & $\mathrm{X}$ & & & & $\mathrm{X}$ & $\mathrm{X}$ \\
\hline Cratogeomys merriami merriami & Pappogeomys merriami $i^{9,7,6}$ & & & & $\mathrm{X}$ & & $\mathrm{X}$ & & & $\mathrm{X}$ & $\mathrm{X}$ & $\mathrm{X}$ \\
\hline Liomys irroratus alleni & & & & & $\mathrm{X}$ & & $\mathrm{X}$ & & & & & $\mathrm{X}$ \\
\hline Urocyon cinereoargenteus nigrirostris & & & & & $\mathrm{X}$ & & $\mathrm{X}$ & & & $\mathrm{X}$ & & $\mathrm{X}$ \\
\hline Mephitis macroura macroura & & & & & $\mathrm{X}$ & & $\mathrm{X}$ & & & $\mathrm{X}$ & & $\mathrm{X}$ \\
\hline Spilogale putorius angustifrons & S. angustifrons ${ }^{9}$ & & & & $\mathrm{X}$ & & $\mathrm{X}$ & & & $\mathrm{X}$ & & $\mathrm{X}$ \\
\hline Bassariscus astutus astutus & & & & & & & $\mathrm{X}$ & & & $\mathrm{X}$ & & $\mathrm{X}$ \\
\hline Mustela frenata frenata & M. frenata perotae 6 & & & & & & $\mathrm{X}$ & & & $\mathrm{X}$ & & $\mathrm{X}$ \\
\hline Sorex saussurei saussurei & & & & & $\mathrm{X}$ & & $\mathrm{X}$ & $\mathrm{X}$ & & $\mathrm{X}$ & $\mathrm{X}$ & $\mathrm{X}$ \\
\hline$* * *$ Sorex ventralis & $\begin{array}{l}\text { S. oreopolus } \\
\text { ventratis }{ }^{7} \text {, S. oreopolus }\end{array}$ & & & & $\mathrm{X}$ & & $\mathrm{X}$ & & & & $\mathrm{X}$ & $\mathrm{X}$ \\
\hline ***Pteronotus parnelli mexicanus & P. parnelli mexicana ${ }^{9}$ & & & & & & & & & $\mathrm{X}$ & & \\
\hline Mormoops megalophylla megalophylla & & & & & $\mathrm{X}$ & $\mathrm{X}$ & $\mathrm{X}$ & & & $\mathrm{X}$ & & $\mathrm{X}$ \\
\hline Leptonycteris curasoae yerbabuenae & L. yerbabuenae ${ }^{9}$, L. sanborni ${ }^{5}$ & & $\mathrm{X}$ & & $\mathrm{X}$ & $\mathrm{X}$ & $\mathrm{X}$ & & & $\mathrm{X}$ & $\mathrm{X}$ & $\mathrm{X}$ \\
\hline ***Leptonycteris nivalis & & & & & & & $\mathrm{X}$ & & & $\mathrm{X}$ & & \\
\hline Anoura geoffroyi lasiopyga & & & & & & $\mathrm{X}$ & $\mathrm{X}$ & & & $\mathrm{X}$ & & $\mathrm{X}$ \\
\hline Choeronycteris mexicana & & & & & $\mathrm{X}$ & $\mathrm{X}$ & $\mathrm{X}$ & & & $\mathrm{X}$ & & $\mathrm{X}$ \\
\hline Tadarida brasiliensis mexicana & & & $\mathrm{X}$ & & $\mathrm{X}$ & $\mathrm{X}$ & $\mathrm{X}$ & & & $\mathrm{X}$ & $\mathrm{X}$ & $\mathrm{X}$ \\
\hline Nyctinomops macrotis & Tadarida macrotis ${ }^{9}$ & & & & $\mathrm{X}$ & $\mathrm{X}$ & $\mathrm{X}$ & & & $\mathrm{X}$ & & $\mathrm{X}$ \\
\hline **Lasiurus blossevillii teliotis & L. borealis teliotis ${ }^{5}$ & & & & & $\mathrm{X}$ & & & & & & $\mathrm{X}$ \\
\hline Lasiurus cinereus cinereus & & & $\mathrm{X}$ & & $\mathrm{X}$ & $\mathrm{X}$ & $\mathrm{X}$ & & & $\mathrm{X}$ & & $\mathrm{X}$ \\
\hline Corynorhinus mexicanus & Plecotus mexicanus ${ }^{9,6,8}$ & & $\mathrm{X}$ & & $\mathrm{X}$ & $\mathrm{X}$ & $\mathrm{X}$ & & & $\mathrm{X}$ & $\mathrm{X}$ & $\mathrm{X}$ \\
\hline Idionycteris phyllotis & Plecotus phyllotis ${ }^{2}$ & & $\mathrm{X}$ & $\mathrm{X}$ & $\mathrm{X}$ & $\mathrm{X}$ & $\mathrm{X}$ & & & & $\mathrm{X}$ & $\mathrm{X}$ \\
\hline Eptesicus fuscus miradorensis & & & & & $\mathrm{X}$ & $\mathrm{X}$ & $\mathrm{X}$ & & & & & $\mathrm{X}$ \\
\hline Myotis velifer velifer & & & & & $\mathrm{X}$ & $\mathrm{X}$ & $\mathrm{X}$ & & & $\mathrm{X}$ & & $\mathrm{X}$ \\
\hline
\end{tabular}


Cuadro 2. Continúa

\begin{tabular}{|c|c|c|c|c|c|c|c|c|c|c|c|c|}
\hline Especie & Sinónimos & 1 & 2 & 3 & 4 & 5 & 6 & 7 & 8 & 9 & 10 & $P E$ \\
\hline ***Myotis occultus & M. lucifugus occultus ${ }^{9}$ & & & & & & & & & $\mathrm{X}$ & & \\
\hline ***Eumops underwoodi sonoriensis & E. u. underwoodii & & & & $\mathrm{X}$ & & & & & $\mathrm{X}$ & & \\
\hline
\end{tabular}

El superíndice en los sinónimos indica la publicación en la que aparecen: 1. Villa-R (1952); 2. Villa-R (1966); 3. Hall (1981); 4. Ceballos y Galindo (1984); 5. Sánchez et al. (1989); 6. Negrete (1991); Negrete y Soberón (1994; 7. Chávez y Ceballos, (1992,1994; 8. Chávez, (1993a, b; 9. Álvarez et al. (1994); 10. Villa y Cervantes, (2003) y PE. Presente estudio

*Registro nuevo; ** Registros publicados pero no listados en los inventarios previos para la reserva; *** Especies no reconocidas por los autores del presente estudio por no encontrar evidencia de su presencia en la zona.

En una recopilación sobre el Pedregal de San Ángel (Rojo, 1994) se publicaron 3 capítulos sobre los mamíferos de esta Reserva. En uno de ellos, basado en una recopilación bibliográfica (Álvarez et al., 1994), se listan 34 especies de mamíferos silvestres (incluyendo las especies exóticas: $R$. rattus y M. musculus). En otro, (Negrete y Soberón 1994) listan 37 especies para esta zona incluyendo Rattus rattus, una de las especies exóticas. Sin embargo, aunque sólo registraron 16 especies en su muestreo, consideran que las especies de mamíferos presentes en la REPSA son 22 y que el resto han sido extirpadas de la zona. En esa misma recopilación, otros autores (Chávez y Ceballos, 1994) registran 24 especies de las 33 originales, basándose en trabajos previos (Villa, 1953; Ceballos y Galindo, 1984). Entre las que anotan como desaparecidas están 1 musaraña Sorex ventralis, 1 tuza (Cratogeomys merriami), 1 ardilla (Spermophilus mexicanus) y 6 especies de ratones (Liomys irroratus, Reithrodontomys fulvescens toltecus, Reithrodontomys megalotis saturatus, Peromyscus maniculatus labecula, Peromyscus melanotis y Microtus mexicanus mexicanus; Negrete, 1991; Chávez Ceballos, 1994; Chávez, 1993b).

Nuestro análisis concluyó que los mamíferos silvestres de la REPSA son 33, con algunas especies distintas a las mencionadas en inventarios previos. Destacan 2 taxa que se registran por primera vez para la REPSA: la ardilla gris ( $S$. aureogaster nigrescens) y el ratón del altiplano $(P$. $m$. melanophrys); 2 más fueron incluidas en este listado: el murciélago colorado Lasiurus blossevillii teliotis, documentado por Sánchez et al. (1989) y el cuinique, S. a. adocetus, documentado por Villa et al. (1991). Esta última especie es endémica de México; sólo estaba registrada para los estados de Jalisco, Morelos, México y Guerrero, pero hay 2 registros más para la parte sur del altiplano mexicano, en el cerro de la Cima, Tlalpan y en Ciudad Universitaria (cercanías del Instituto de Biología), y 3 para los estados de Hidalgo y Tlaxcala (Hall, 1981). Sin embargo, otros autores (Valdez y Ceballos, 2005) mencionan que podrían ser mascotas liberadas porque no hay evidencia de poblaciones silvestres.

Asimismo, nuestros resultados no reconocen la presencia de 8 especies registradas en publicaciones previas (Cuadro 2). Estos registros anecdóticos (el sinónimo y referencia, entre paréntesis en la lista) se refieren a Sorex ventralis (Sorex oreopolus ventralis, en Ceballos, 1984 y Villa y Cervantes, 2003), Microtus mexicanus mexicanus, Spermophilus mexicanus mexicanus (Ceballos y Galindo, 1984; Álvarez et al., 1994, Negrete, 1991; Negrete y Soberón, 1994) y Peromyscus melanotis (Ceballos y Galindo, 1984; Negrete, 1991; Negrete y Soberón, 1994). Además de no haber encontrado ningún ejemplar para estas especies en la colección (CNMA) que ayudara a corroborar su presencia, los roedores mencionados han sido registrados en asociaciones vegetales diferentes a las de la REPSA.

Por otro lado, de acuerdo a una revisión reciente de las musarañas que se distribuyen en México (Carraway, 2007), en la REPSA hay 3 especies, S. s. saussurei (CNMA 9059), S. veraecrucis altoensis (CNMA 9059, nueva subespecie) y S. ventralis (ENCB 5229). Sin embargo, en el presente trabajo no fueron incluidos estos cambios taxonómicos, ya que uno de los ejemplares no tiene cráneo y el otro está incompleto, y los principales caracteres diagnósticos en los que se basan están en los incisivos y el cráneo.

Eumops underwoodi, registrada en el Pedregal de San Francisco (sic), zona muy cercana a Ciudad Universitaria, fue otra de las especies cuyo ejemplar de referencia tampoco se encontró (UAMI), razón por la cual en una publicación previa, Sánchez, (1989) menciona que el registro más cercano a la ciudad de México se encuentra en Palo Bolero, Morelos.

Pteronotus parnellii mexicanus, Leptonycteris nivalis (Álvarez, 1994; Negrete y Soberón, 1994) y Myotis occultus (Myotis lucifugus occultus, en Álvarez et al. ,1994) son registros que se encuentran en el Distrito Federal, pero no en la Reserva y en este trabajo solamente se consideraron 
los registros reales, no los potenciales.

Por lo tanto, al comparar la lista de 33 especies obtenida en este estudio con las listas anteriores, encontramos que solamente 24 especies coinciden. Esto fundamenta la importancia que tiene el que los registros se encuentren apropiadamente documentados y bajo el resguardo de una colección científica para su consulta y verificación en cualquier momento.

Otra información de relevancia surgida de este trabajo fue conocer los registros por especie y la fecha de recolecta de los ejemplares depositados en las colecciones. Se documentaron especies cuyo último registro es de 20-45 años atrás. Entre las especies con registros únicos están Idionycteris phyllotis de 1962, Eptesicus fuscus miradorensis de 1968, Myotis v. velifer de 1975, Mustela f. frenata de 1966, Cratogeomys m. merriami de 1970, Spermophilus a. adocetus de 1974 y Peromyscus m. melanophrys de 1984. Otras especies, sólo tienen 2 registros: Corynorhynus mexicanus de 1962 y Sigmodon hispidus de 1972, y los roedores Baiomys taylori analogus y Liomys irroratus alleni, aunque están bien documentados (69 y 31 ejemplares), sus últimos registros son de 1963 y 1964, respectivamente.

En este mismo caso se encuentran la zorra gris ( $U$. cinereoargenteus nigrirostris) y el conejo castellano $(S$. floridanus orizabae), cuyo último registro en colecciones corresponde a 1981, es decir, con 26 años de antigüedad. Sin embargo, es importante destacar que en fechas recientes se documentó la presencia de ambos taxa por medio de fotografías, registros visuales y excrementos. Adicionalmente, para la zorra se realizó un estudio con radio telemetría (García, 2007) y en la CNMA está el registro fotográfico de un ejemplar que se encontró muerto, en diciembre de 2006, el cual se recolectó y se entregó al Museo de Zoología de la Facultad de Ciencias. El cacomixtle, Bassariscus astutus se había documentado sólo por observación, huellas y excrementos; el primer ejemplar documentado en colecciones se obtuvo durante la realización del presente estudio (2004), además de registrarse su presencia durante la realización de estudios sobre área de actividad y uso de hábitat (Castellanos y List, 2005 y Castellanos, 2006).

Por lo tanto, el número de especies con registros exclusivamente antiguos, disminuyó por las recolecciones obtenidas en el presente trabajo, principalmente con la recuperación de individuos muertos. En este caso, además del cacomixtle y la zorra, están el zorrillo, Spilogale putorius angustifrons, cuyo último registro era de 1978 y el ratón, Reithrodontomys fulvescens toltecus, que se colectó en 1990. En un trabajo previo (Negrete y Soberón, 1994), está ultima especie, al igual que los roedores Reithrodontomys megalotis saturatus, S. hispidus berlandieri, L. irroratus alleni y la tuza C. m. merriami fueron considerados como ausentes de la REPSA.

De los 628 registros obtenidos en el presente estudio, $340(50 \%)$ corresponden a zonas que colindan con la Reserva, donde las recolectas más antiguas corresponden a 1943 y las más recientes a 1991. Estas áreas colindantes con la REPSA han sido ocupadas casi en su totalidad por asentamientos humanos, con la consecuente desaparición de la fauna silvestre (Cuadro 3). Por lo tanto, es probable que muchas de estas especies sólo se encuentren al interior

Cuadro 3. Puntos geográficos donde se encuentran los registros de los mamíferos de la Reserva Ecológica del Pedregal de San Ángel en Ciudad Universitaria y sus alrededores

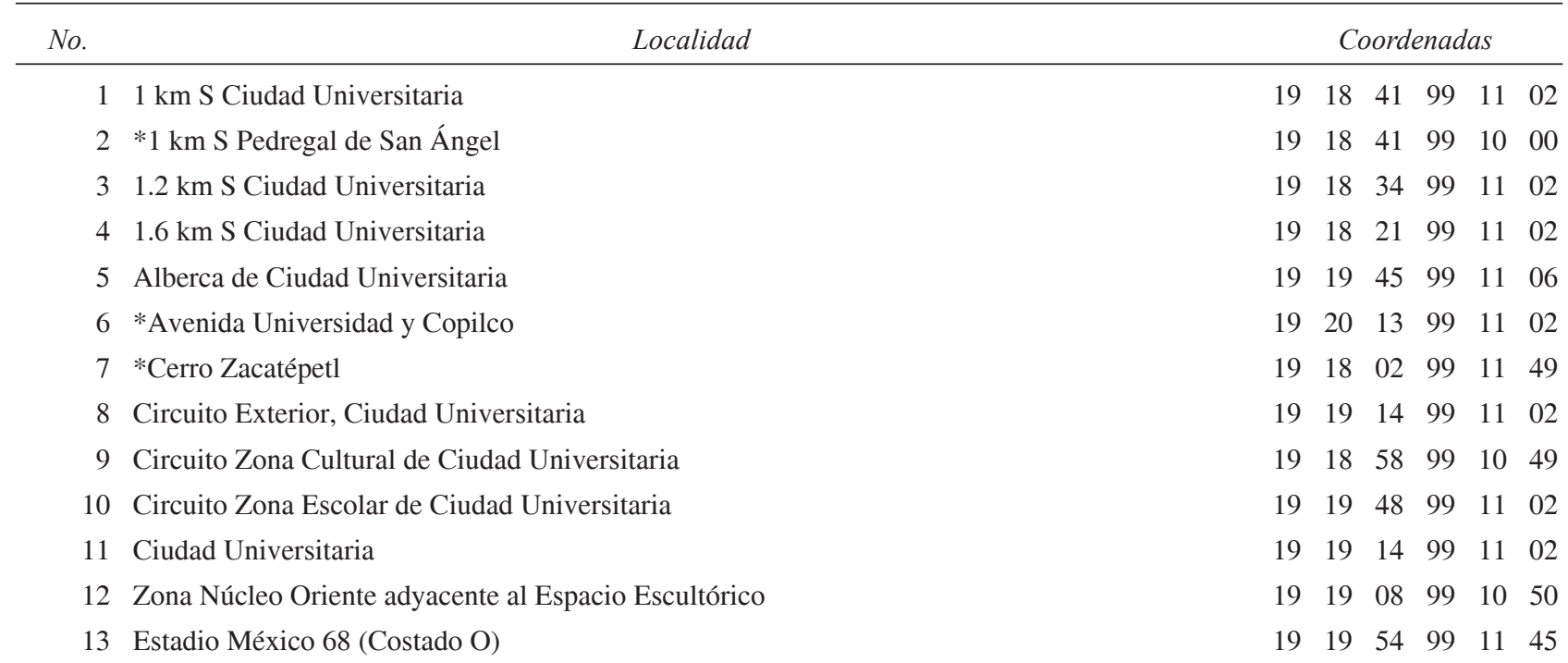


Cuadro 3. Continúa

\begin{tabular}{|c|c|c|c|c|c|c|c|}
\hline \multirow{2}{*}{$\frac{\text { No. }}{14}$} & \multirow{2}{*}{$\begin{array}{lr} & \text { Localidad } \\
\text { Estadio México 68, (Lateral Insurgentes) } & \end{array}$} & \multicolumn{6}{|c|}{ Coordenadas } \\
\hline & & 19 & 19 & 53 & 99 & 11 & 23 \\
\hline 15 & Facultad de Ciencias (Edificio Antiguo), Ciudad Universitaria & 19 & 19 & 58 & 99 & 10 & 57 \\
\hline 16 & Facultad de Ciencias, Ciudad Universitaria & 19 & 192 & 28 & 99 & 10 & 53 \\
\hline 17 & Facultad de Ingeniería, Ciudad Universitaria & 19 & 19 & 39 & 99 & 10 & 48 \\
\hline 18 & Facultad de Medicina, Ciudad Universitaria & 19 & 19 & 55 & 99 & 10 & 43 \\
\hline 19 & Facultad de Odontología & 19 & 192 & 20 & 99 & 10 & 52 \\
\hline 20 & *Falda SO Cerro Zacatépetl, 3.9 km SO Monumento Álvaro Obregón, Del. Álvaro Obregón. & 19 & 19 & 14 & 99 & 12 & 49 \\
\hline 21 & Frontón Cerrado, Ciudad Universitaria & 19 & 19 & 26 & 99 & 11 & 11 \\
\hline 22 & Instituto de Biología (Circuito Exterior), Ciudad Universitaria & 19 & 19 & 12 & 99 & 11 & 33 \\
\hline 23 & Invernadero Faustino Miranda, Instituto de Biología & 19 & 19 & 44 & 99 & 10 & 49 \\
\hline 24 & Jardín Botánico Exterior,Instituto de Biología & 19 & 19 & 06 & 99 & 11 & 36 \\
\hline 25 & Límite E Ciudad Universitaria & 19 & 19 & 13 & 99 & 10 & 19 \\
\hline 26 & Límite S Ciudad Universitaria & 19 & 18 & 26 & 99 & 11 & 05 \\
\hline 27 & Límite SE Ciudad Universitaria & 19 & 18 & 29 & 99 & 10 & 24 \\
\hline 28 & Límite SSE Ciudad Universitaria & 19 & 18 & 31 & 99 & 10 & 33 \\
\hline 29 & Límite SSO Ciudad Universitaria & 19 & 18 & 32 & 99 & 11 & 27 \\
\hline 30 & Zona Núcleo Poniente adyacente a la Mesa Vibradora & 19 & 18 & 58 & 99 & 11 & 47 \\
\hline 31 & Museo UNIVERSUM & 19 & 18 & 40 & 99 & 10 & 59 \\
\hline 32 & *Pedregal de San Ángel, Del. Álvaro Obregón & 19 & 19 & 14 & 99 & 10 & 00 \\
\hline 33 & *Pedregal de San Ángel, 0.80 km S Tizapán, Del. Álvaro Obregón & 19 & 19 & 35 & 99 & 12 & 14 \\
\hline 34 & *Pedregal de San Ángel, 1.61 km S Tizapán, Del Álvaro Obregón & 19 & 19 & 09 & 99 & 12 & 14 \\
\hline 35 & *Pedregal de San Ángel, 1.61 km S=Monumento Álvaro Obregón- & 19 & 20 & 07 & 99 & 11 & 47 \\
\hline 36 & *Pedregal de San Ángel, 1.61 km SO Tizapán, Del Álvaro Obregón & 19 & 19 & 24 & 99 & 12 & 52 \\
\hline 37 & Pedregal de San Ángel, 2.09 km S Monumento Álvaro Obregón. & 19 & 19 & 36 & 99 & 11 & 09 \\
\hline 38 & *Pedregal de San Ángel, 3.06 km SO Monumento Álvaro Obregón, Del. Álvaro Obregón. & 19 & 19 & 34 & 99 & 12 & 22 \\
\hline 39 & Pedregal de San Ángel, 3.22 km S Monumento Álvaro Obregón, Del. Álvaro Obregón. & 19 & 18 & 59 & 99 & 11 & 09 \\
\hline 40 & Pedregal de San Ángel, 3.70 km S Monumento Álvaro Obregón, Del Álvaro Obregón & 19 & 18 & 43 & 99 & 11 & 09 \\
\hline 41 & Pedregal de San Ángel, 4.18 km S Monumento Álvaro Obregón, Del. Álvaro Obregón. & 19 & 18 & 28 & 99 & 11 & 09 \\
\hline 42 & *Pedregal de San Ángel, 6.84 km SOÁlvaro Obregón, Del. Álvaro Obregón. & 19 & 21 & 21 & 99 & 14 & \\
\hline 43 & Pedregal de San Jerónimo & 19 & 19 & 24 & 99 & 13 & 00 \\
\hline 44 & *Pedregal, 1.61 km S Coyoacán (Pedregal de San Francisco) & 19 & 20 & 07 & 99 & 09 & 42 \\
\hline 45 & *Presa Anzaldo, Pedregal de San Ángel & 19 & 18 & 32 & 99 & 13 & 10 \\
\hline 46 & Rectoría, Ciudad Universitaria & 19 & 19 & 54 & 99 & 11 & 18 \\
\hline 47 & Reserva Ecológica del Pedregal de San Ángel de Ciudad Universitaria & 19 & 18 & 45 & 99 & 11 & 17 \\
\hline 48 & *San Ángel Jardines del Pedregal, Del. Álvaro Obregón. & 19 & 19 & 11 & 99 & 12 & 10 \\
\hline 49 & *San Jerónimo, 6.44 km SO Álvaro Obregón Del. Álvaro Obregón. & 19 & 16 & 30 & 99 & 14 & 45 \\
\hline 50 & San Jerónimo, 7.24km SOÁlvaro Obregón, Del. Álvaro Obregón. & 19 & 21 & 12 & 99 & 15 & 06 \\
\hline 51 & $\begin{array}{l}\text { Senda Ecológica UNIVERSUM, Zona de amortiguamiento A4, Reserva Ecológica del Pedregal } \\
\text { de San Angel, Ciudad Universitaria. }\end{array}$ & 19 & 18 & 46 & 99 & 10 & \\
\hline 52 & Unidad de seminarios Dr. Ignacio Chávez, Ciudad Universitaria & 19 & 18 & 48 & 99 & 11 & \\
\hline
\end{tabular}

*Localidades en los alrededores de la REPSA y Ciudad Universitaria. 


\section{de la REPSA.}

Del total de los mamíferos terrestres registrados para el Distrito Federal (Ramírez-Pulido 1986) la REPSA tiene el $75 \%$ de los órdenes, el $88 \%$ de las familias, el $61 \%$ de los géneros y el $52 \%$ de las especies. Respecto a la Cuenca de México (López-Forment, 1989), el área de estudio cuenta con el $75 \%$ de los órdenes, el $83 \%$ de las familias, el $67 \%$ de los géneros y el $39 \%$ de las especies. Respecto al país (Ramírez-Pulido et al., 2005), tiene el 50\% de los órdenes, el $44 \%$ de las familias, el $17 \%$ de los géneros y el $7 \%$ de las especies. Sin duda, si consideramos además la extensión de la zona (237 ha), estas cifras muestran la gran riqueza de especies de mamíferos silvestres de la REPSA.

Es importante resaltar la presencia de especies endémicas de México en la REPSA, de las monotípicas y de las incluidas en categorías de riesgo de la NOM-059 (Leptonycteris curasoae yerbabuenae, Choeronycteris mexicana, Myotis velifir velifer (Cuadro 1)). La Reserva es uno de los últimos reductos de material genético de especies cuya localidad tipo se encuentra en la Cuenca de México, lo que pone de manifiesto su relevancia como hábitat de los mamíferos de esta Cuenca, particularmente los de la parte sur. Por lo tanto, la información derivada de su componente mastofaunístico imprime a la REPSA un alto valor natural además de sus características ecológicas particulares.

En este trabajo se aporta información documentada y actualizada de las diferentes especies de mamíferos que se encuentran en la REPSA, se confirma la presencia de algunas especies y se desconocen registros publicados que carecen de evidencia para verificar su existencia pasada o presente; asimismo, se agregan nuevos registros. Por otro lado, los resultados ponen de manifiesto la importancia de las colecciones biológicas, de las revisiones adecuadas de la literatura, así como del monitoreo periódico de las poblaciones silvestres de mamíferos para documentar su presencia en áreas protegidas.

Este componente mastofaunístico es importante para el mantenimiento de la biodiversidad de la zona, por lo que debe continuar su protección y estudio, particularmente por tratarse de uno de los últimos reductos del ecosistema natural al interior de la Ciudad de México.

\section{Agradecimientos}

Imelda Marín, Helxine Fuentes y Raúl Martínez auxiliaron en el trabajo de campo y gabinete, Jesús Fernández y dos revisores anónimos aportaron valiosos comentarios al manuscrito Armando Peralta proporcionó la imagen digitalizada de la REPSA y Gibran Hoffmann auxilió en la edición digitalizada. Las recolectas se realizaron al amparo del permiso FAUT002 expedido por la SEMARNAT. Javier Caballero y Antonio Lot dieron autorización para realizar trabajo de campo en el Jardín Botánico, Instituto de Biología, UNAM, y al interior de la REPSA, respectivamente. Los curadores de las colecciones mastozoológicas consultadas, amablemente proporcionaron información sobre los ejemplares de museo bajo su custodia, particularmente José Ramírez, Juan Carlos López, Livia León, Robert A. Timm y James Patton. La Unidad de Informática para la Biodiversidad (UNIBIO), Instituto de Biología, UNAM, financió este proyecto como parte del megaproyecto universitario del Sistema de Informática para la Biodiversidad y el Ambiente (SIBA), del programa Investigación Multidisciplinaria de Proyectos Universitarios de Liderazgo Académico (IMPULSA).

\section{Literatura citada}

Álvarez, F. J., J. Carabias L., J. Meave del Castillo, P. Moreno Casasola, D. Nava Fernández, F. Rodríguez Zahar, C. Tovar González y A. Valiente-Banuet. 1994. Proyecto para la creación de una reserva en el Pedregal de San Ángel. In Reserva Ecológica "El Pedregal de San Ángel”: ecología, historia natural y manejo, A. Rojo (ed.) Universidad Nacional Autónoma de México, México, D. F. p. 343-353.

Álvarez, T., S. T. Álvarez-Castañeda y M. González-Escamilla. 1997. Localidades típicas de mamíferos terrestres en México. Centro de Investigaciones Biológicas del Noroeste, Escuela Nacional de Ciencias Biológicas, Instituto Politécnico Nacional, México, D. F. 175 p.

Baker, R. H. 1952. Geographic range of Peromyscus melanophrys, with description of a new subspecies. University of Kansas Publications Museum of Natural History. 5:251-258.

Carraway, L. N. 2007. Shrews (Eulypotyphla: Soricidae) of México. Monographs of the Western North American Naturalist 3:1-91.

Castellanos, M. G. 2006. Sobre el ámbito hogareño y los hábitos alimentarios de un carnívoro en un ambiente suburbano. El cacomixtle (Bassariscus astutus) en la Reserva Ecológica "El Pedregal de San Ángel”, Ciudad Universitaria, México, D. F." Tesis, Facultad de Ciencias, Universidad Nacional Autónoma de México, México, D. F. 97 p.

Castellanos, M. G. y R. List. 2005. Área de actividad y uso de hábitat del cacomixtle, (Bassariscus astutus) en el "El Pedregal de San Ángel”. Revista Mexicana de Mastozoología 9:113-122.

Ceballos, G. y C. Galindo. 1984. Mamíferos silvestres de la Cuenca de México. Limusa/ MAB, México, D. F. 299 p.

Chávez, J. C. y G. Ceballos. 1992. Los mamíferos silvestres del Pedregal. Oikos 13:4.

Chávez, J. C. 1993a. Dinámica poblacional y uso de hábitat por roedores en un matorral de palo loco (Senecio praecox). Tesis, Facultad de Ciencias, Universidad Nacional Autónoma de México, México, D. F. 63 p. 
Chávez, J. C. 1993b. Los roedores silvestres de El Pedregal. Oikos 21:4

Chávez, J. C. y G. Ceballos. 1994. Historia natural comparada de los pequeños mamíferos de la Reserva El Pedregal. In Reserva Ecológica "El Pedregal de San Ángel": ecología, historia natural y manejo", A. Rojo (eds.). Universidad Nacional Autónoma de México, México, D. F. p. 229-238.

de la Fuente, J. R. 2005. Acuerdo por el que se rezonifica, delimita e incrementa la zona de la Reserva Ecológica del Pedregal de San Ángel de Ciudad Universitaria. Gaceta UNAM, 3813:19-21.

Darwin Core V. 3.0. Portal. http://darwincore.calacademy.org/

García, P. M. N. 2007. Sobre el ámbito hogareño y los hábitos alimentarios de un carnívoro en un ambiente suburbano. La zorra gris (Urocyon cinereoargenteus) en la Reserva Ecológica "El Pedregal de San Ángel”. Ciudad Universitaria. México, D. F. Tesis, Facultad de Ciencias, Universidad Nacional Autónoma de México 90 p.

Hafner, S. M., W. L. Gannon, J. Salazar-Bravo y S. T. Álvarez Castañeda. 1997. Mammal collections in the Western Hemisphere. American Society of Mammalogists. Allen, Lawrence, Kansas. 93 p.

Hafner, J. C., J. E. Light, D. J. Hafner, M. S. Hafner, E. Reddington, D. S. Rogers y B. R. Riddle. 2007. Basal clades and molecular systematics of heteromyid rodents. Journal of Mammalogy 88:1129-1145.

Hall, E. R. 1981. The mammals of North America . Wiley \& Sons, Hoboken, New Jersey. 1181 p.

Hall, E. R y K. R. Kelson.1959. The mammals of North America. Ronald, New York, vol. 1, 1-546+79 p; vol. 2, 547-1083 p.

Herrera, T., E. Pérez-Silva y V. H. Valenzuela. 2006. Nueva contribución al conocimiento de los macromicetos de la Reserva Ecológica del Pedregal de San Ángel, D. F., México. Revista Mexicana de Biodiversidad 77:51-57.

INEGI (Instituto Nacional de Estadística, Geografía e Informática). 2001. Carta topográfica Ciudad de México, 1:50 000 (E14A39). Dirección General de Geografía. Instituto Nacional de Estadística, Geografía e Informática, Aguascalientes, Aguascalientes.

López- Forment, C. W. 1989. La situación actual de los mamíferos en el Valle de México. In Ecología urbana, Gio-Argaéz, R, Hernández, R. I. y E. Saínz-Hernández (eds.) México, D. F. 167-170 p.

Medellín, R. A., H. T. Arita y O. H. Sánchez. 1997. Identificación de los murciélagos de México. Clave de campo, Publicaciones especiales Número 2. Asociación Mexicana de Mastozoología, México. D. F. 83 p.

Negrete, Y. A. 1991. Los mamíferos silvestres de la Reserva Ecológica "El Pedregal". Tesis, Facultad de Ciencias, Universidad Nacional Autónoma de México, México, D. F. $114 \mathrm{p}$.

Negrete, Y. A. y J. Soberón. 1994. Los mamíferos silvestres de la Reserva Ecológica El Pedregal de San Ángel. In Reserva Ecológica "El Pedregal de San Ángel”: ecología, historia natural y manejo, A. Rojo (eds.), Universidad Nacional Autónoma de México, México, D. F. p. 219-228.

Peralta-Higuera A., J. Prado-Molina, E. Cabral-Cano y O. DíazMolina 2005 . Mapa de la Reserva Ecológica del Pedregal de
San Ángel en Ciudad Universitaria, escala 1: 2500, Instituto de Geografía e Instituto de Geofísica, UNAM. Gaceta UNAM, 2 de junio de 2005.

Prado Molina J., y A. Peralta-Higuera. 2005. Anexo técnico perteneciente al Acuerdo mediante el cual se rezonifica, delimita e incrementa la Reserva Ecológica del Pedregal de San Ángel en Ciudad Universitaria. Comité Técnico de la Reserva Ecológica del Pedregal de San Ángel en Ciudad Universitaria. Gaceta UNAM, 2 de junio, 19 p.

Ramírez-Pulido, J., M. C. Britton, A. Perdomo y A. Castro. 1986. Guía de los mamíferos de México. Universidad Autónoma Metropolitana, unidad Iztapalapa, México, D. F., 720 p.

Ramírez-Pulido, J., J. Arroyo-Cabrales, A. Castro-Campillo. 2005. Estado actual y relación nomenclatural de los mamíferos terrestres de México. Acta Zoologica Mexicana (n. s.) 21:21-82.

Rojo, C. A. 1993. Plan de manejo. Reserva Ecológica "El Pedregal de San Ángel". In Reserva Ecológica "El Pedregal de San Ángel": ecología, historia natural y manejo, A. Rojo (eds.), Universidad Nacional Autónoma de México, México, D. F. p. 371-382.

Rojo, C. A. 1994. Reserva Ecológica "El Pedregal de San Ángel”: ecología, historia natural y manejo, A. Rojo (eds.), Universidad Nacional Autónoma de México, México, D. F. $410 \mathrm{p}$.

Romero-Almaraz, M. de L., C. Sánchez-Hernández, C. GarcíaEstrada y R. D. Owen. 2000. Mamíferos pequeños: manual de técnicas de captura, preparación, preservación y estudio. Facultad de Ciencias,UNAM/Instituto de Biología, UNAM/ Centro de Investigaciones Biológicas, Universidad Autónoma del Estado de Morelos. México, D.F. 151 p.

Sánchez-H, O., G. López-Ortega, R. López-Wilchis. 1989. Murciélagos de la ciudad de México y sus alrededores. In Ecología urbana, R. Gio-Argaéz, , R. I. Hernández, y E. Saínz-Hernández (eds.), Sociedad Méxicana de Historia Natural, México, D. F. p. 141-165.

SCT (Secretaría de Comunicaciones y Transportes). 1987. Carta topográfica del Distrito Federal, escala 1:100 000. México, D. F.

SEMARNAT (Secretaría de Medio Ambiente y Recursos Naturales). 2002. Norma Oficial Mexicana. NOM-059ECOL-2001. Protección ambiental - Especies nativas de México de flora y fauna silvestres - Categorías de riesgo y especificaciones para su inclusión, exclusión o cambio Lista de especies en riesgo. Diario Oficial de la Federación, Segunda sección, Mamíferos, miércoles 6 de marzo, p.136147.

UNIBIO (Unidad de Informática para la Biodiversidad). 2006.Instituto de Biología, Universidad Nacional Autónoma de México. http://unibio.ibiologia.unam.mx Valdez A. M. y G. Ceballos, 2005. Spermophilus adocetus (Merriam, 1903). In Los Mamíferos Silvestres de México, Ceballos, G. y G. Oliva (eds.). Comisión Nacional para el Conocimiento y Uso de la Biodiversidad/Fondo de Cultura Económica, México, D. F. p. 559-560.

Villa-R, B. 1952. Mamíferos silvestres del Valle de México. Anales del Instituto de Biología, Universidad Nacional Autónoma de México. 23:269-492. 
Villa-R, B. 1966. Los murciélagos de México. Su importancia en la economía y la salubridad-Su clasificación sistemática. Instituto de Biología, UNAM, México, D. F. 491 p.

Villa, R. B. y F. A. Cervantes. 2003. Los mamíferos de México. Iberoamérica/Instituto de Biología, UNAM, México, D. F. 140 p.

Villa, R. B y V. Sánchez C. 1991. La ardilla terrestre o cuiniqui
Spermophilus adocetus Merriam,1905 (Rodentia:Sciuridae) en la región central de México y su status taxonómico. Anales del Instituto de Biología, Universidad Nacional Autónoma de México, Serie Zoología 62:511-519.

Wieczorek, J. 2001. MaNIS/HerpNet/ORNIS Georeferencing Guidelines. University of California, Berkerleley. http:// manis.mvz.berkeley.edu/

Apéndice 1. Ejemplares de museo que documentan la presencia de mamíferos de la Reserva Ecológica del Pedregal de San Ángel,„Ciudad Universitaria, Universidad Nacional Autónoma de México, México, D.F. Se indica el nombre común y científico de la especie, el número total de ejemplares por especie (en paréntesis), seguido de la colección en la que están depositados y el número de catálogo correspondiente. Nomenclatura científica y orden filogenético, de acuerdo con Ramírez-Pulido (2005), excepto la familia Heteromyidae para la cual se siguió a Hafner et al. (2007).

Acrónimos: CNMA = Colección Nacional de Mamíferos, Instituto de Biología, UNAM. ENCB = Colección de Cordados, Escuela Nacional de Ciencias Biológicas, Instituto Politécnico Nacional. MZFC = Museo de Zoología "Alfonso L. Herrera", Facultad de Ciencias, UNAM. UAMI = Universidad Autónoma Metropolitana, Iztapalapa. KU = Natural History Museum, University of Kansas. FMNH $=$ Field Museum of Natural History. UMMZ = Museum of Zoology, University of Michigan. MVZ = Museum of Vertebrate Zoology, University of California, Berkeley.

\begin{tabular}{|c|c|}
\hline Tlacuache & nero \\
\hline $\begin{array}{l}\text { Didelphis virginiana californica (41): CNMA 437, 796, 3785- } \\
\text { 3787, 8736, 11305, 16491, 23070, 30722-30723, 31342, 33417, } \\
\text { 34642-34645, 34780, 34858, 37131, 40199, 42908-42910, } \\
\text { 43372-43377, 44049-44054,44085. ENCB 40020, 40730. } \\
\text { MZFC 3465, 3468. }\end{array}$ & $\begin{array}{l}\text { Peromyscus gratus gratus (316): CNMA 689-696, 698-699, } \\
\text { 713-731, 797-798, 3804, 9479,10113-10122,11624-11625, } \\
\text { 11646-11676, 14863, 14864, 15208-15209, 15123-15124, } \\
\text { 15608, 15650, 33517-33525, 33591, 34650-34676, 42916, } \\
\text { 44071-44082. KU 28181-28186, 28191-28193, 28212-28228, } \\
\text { 28230. UAMI: 2788-2789. UMMZ 89093-89117, 89119-89132, }\end{array}$ \\
\hline stellano & $\begin{array}{l}\text { 89135, 89138-89155, 89157, 89159-89165, 89407, 90714, } \\
\text { 93442-93443. MVZ: 100510-100541. FMNH 55833-55848, } \\
\text { 55861-55865, 55968. MZFC 184-186. ENCB 26, 29, 182-190, } \\
\text { 193-196, 204-205, 574-580, 694. }\end{array}$ \\
\hline $\begin{array}{l}\text { eogaster nigrescens (4): CNMA 39693, 42915, } \\
2 .\end{array}$ & a \\
\hline $\begin{array}{l}\text { Cuinique } \\
\text { Spermophilus adocett }\end{array}$ & \\
\hline $\begin{array}{l}\text { Ardillón, ardilla del pedregal, ardilla de las rocas, tecachalote } \\
\text { Spermophilus variegatus variegatus (24): CNMA 8303, } \\
\text { 15643,15644, 15588-15589, 16006, 16487, 17040, 27271, } \\
\text { 27693, 34648-34649, 39694, 42914, 43378-43380, 44070. } \\
\text { MZFC 189, 462, 5640. ENCB 787, 2018, } 10250 .\end{array}$ & $\begin{array}{l}\text { nys fulvescens toltecus (20): CNMA 542, 693, } \\
\text { 34679, 44083-44084. UMMZ 88810-88811, } \\
\text { 95924-95926. FMNH 55917. ENCB 21, 28, } 351\end{array}$ \\
\hline Ratón & \\
\hline $\begin{array}{l}\text { Baiomys taylori analogus (69): CNMA 200, 588, 602. KU } \\
\text { 28076, 28096- } \\
\text { 28099. FMNH 55928-55941, 55969-55970. ENCB 27, 198-19 } \\
\text { 201-202, 692. MVZ 100329-100330. UMMZ 88909-88944, }\end{array}$ & $\begin{array}{l}\text { Ratón silvestre } \\
\text { Reithrodontomys megalotis saturatus (9): CNMA 543-545. KU } \\
\text { 28063-28064. UMMZ 88809, 88812. FMNH 61830-61831. }\end{array}$ \\
\hline & $\begin{array}{l}\text { a hispida } \\
\text { idus (2): ENCB 5873-5874. }\end{array}$ \\
\hline $\begin{array}{l}\text { Neotoma mexicana torquata (11): CNMA 5471-5472, } 15223 \text {, } \\
\text { 34680-34682. ENCB 22-25, 5182. }\end{array}$ & Cratogeomys merriami merriami (1): CNMA 15118. \\
\hline
\end{tabular}


Ratón espinoso mexicano

Heteromys irroratus alleni (31): CNMA 4584,11549. FMNH 55791-55792. UMMZ 88748-88774.

Zorra gris

Urocyon cinereoargenteus nigrirostris (1): CNMA 15636.

Comadreja

Mustela frenata frenata (1): CNMA 9623.

Zorrillo encapuchado

Mephitis macroura macroura (3): CNMA 14592, 15634,16887.

Zorrillo manchado

Spilogale putorius angustifrons (5): CNMA 16885, 44069.

ENCB 520, 5875-5876.

Cacomixtle

Bassariscus astutus astutus (4): CNMA 42913, 43383, 44067,44068

Musaraña

Sorex saussurei saussurei (18): CNMA 9059, 38637, 42911-

42912, 43368-43371, 44055-44062. ENCB 5229. UMMZ

88639 .

Murciélago bigotudo de cara plegada

Mormoops megalophylla megalophylla (2): CNMA 3928, 9886.

Murciélago magueyero

Leptonycteris curasoae yerbabuenae (4): CNMA 4729, 34647, 42770-42771.
Murciélago lenguilargo

Anoura geoffroyi lasiopyga (6): CNMA 15478, 16965, 22570, 34646, 42768-42769.

Murciélago trompudo

Choeronycteris mexicana (14): CNMA 15485-15486, 4276042767, 44063-44066.

Murciélago de cola libre, murciélago guanero mexicano Tadarida brasiliensis mexicana (13): CNMA 1747, 3922, 9874, 11495, 13445, 14878, 15580, 15642, 17035,18531. UAMI: $1622,4857,10411$

Murciélago coludo de orejas grandes, murciélago de cola libre Nyctinomops macrotis (6): CNMA 11497, 39349, 40871-40873. MZFC 6685.

Murciélago colorado

Lasiurus blossevilli teliotis (1) CNMA 18531

Murciélago escarchado o canoso

Lasiurus cinereus cinereus (2) CNMA 10682, UMMZ 91904.

Murciélago orejas de mula

Corynorhinus mexicanus (2): CNMA 7358, 9718.

Murciélago de cuatro orejas

Idionycteris phyllotis (1): CNMA 6145

Gran murciélago moreno

Eptesicus fuscus miradorensis (1): ENCB 3730.

Murcielaguito pardo

Myotis velifer velifer (1): ENCB 7641. 